\title{
AN INTERPRETATION OF STOCHASTIC DIFFERENTIAL EQUATIONS AS ORDINARY DIFFERENTIAL EQUATIONS WHICH DEPEND ON THE SAMPLE POINT
}

\author{
BY HÉCTOR J. SUSSMANN ${ }^{1}$
}

Communicated by Daniel Stroock, September 27, 1976

We consider: (I) a "deterministic" ordinary differential equation

$$
d x(t)=f(x(t)) d t+g(x(t)) d w(t), \quad x \in \mathbf{R}^{n},
$$

where $f, g$ are vector fields in $\mathbf{R}^{n}$ and $w:[a, b] \rightarrow \mathbf{R}$ is a continuous function, and (II) the stochastic equation

$$
d X=f(X) d t+g(X) d W
$$

where $f, g$ are as before, and $W=\left\{W_{t}: t \in[a, b]\right\}$ is a stochastic process with continuous sample paths, defined on a probability space $P=(\Omega, A, P)$.

In order to define what is meant by a solution of (stoch), the most obvious approach would go as follows: a process $\left\{X_{t}: t \in[a, b]\right\}$ is said to be a solution of (stoch) if, for each sample point $\omega \in \Omega$, the function $t \rightarrow X_{t}(\omega)$ is a solution of

$\left(\operatorname{det}_{\omega}\right)$

$$
d x(t)=f(x(t)) d t+g(x(t)) d w_{\omega}(t),
$$

where $w_{\omega}(t)=w_{t}(\omega)$. Usually, this approach is not followed because of the technical difficulties that appear when one tries to solve (det) for arbitrary continuous $w$. One is therefore forced to use other lines of attack, and to study (stoch) directly. This gives rise to at least two nonequivalent theories, namely, the one due to Ito, and that of Fisk and Stratonovich.

The purpose of this note is to announce that the "obvious approach" described in the beginning of the preceding paragraph can actually be pursued all the way, leading to a simple construction of solutions of (stoch) for arbitrary processes $W$ with continuous sample paths. When $W$ is a Wiener process, our construction gives the same result as the ordinary solution in the sense of Stratonovich.

First, let us define what is meant by a solution of (det) when $w$ is only continuous. We say that a curve $x: t \rightarrow x(t), a \leqslant t \leqslant b$, is a solution of (det), if there exists a neighborhood $U$ of $w$ in the space $C^{0}([a, b], \mathbf{R})$ of continuous real-valued functions on $[a, b]$ (with the sup norm), and a continuous map $\Gamma$ : $U \rightarrow C^{0}\left([a, b], \mathbf{R}^{n}\right)$, such that:

AMS (MOS) subject classifications (1970). Primary 60H10; Secondary 34F05.

${ }^{1}$ Research partially supported by NSF Grant GP-37488. 
(i) $\Gamma(w)$ is the given curve $x$, and

(ii) whenever the function $\bar{w} \in U$ is of class $C^{1}$, then the curve $\Gamma(\bar{w})$ is a solution of

$$
\frac{d x}{d t}(t)=f(x(t))+\frac{d \bar{w}}{d t}(t) g(x(t))
$$

in the ordinary sense.

THEOREM 1. Assume that

(1) $f$ is locally Lipschitz,

(2) $g$ is $C^{1}$ with locally Lipschitz first partial derivatives,

(3) there is a constant $K$ such that $|f(x)| \leqslant K(1+|x|)$ for all $x$ in $\mathbf{R}^{n}$, and

(4) the partial derivatives of the components of $g$ are uniformly bounded.

Then for every $w \in C^{0}([a, b], \mathbf{R})$ and every $x_{0} \in \mathbf{R}^{n}, t_{0} \in[a, b]$, there exists one and only one solution $x$ of (det) such that $x\left(t_{0}\right)=x_{0}$. Moreover, the solution $x$ depends continuously on $t_{0}, x_{0}$ and $w$.

The proof of this theorem is not hard. Here is a brief sketch. When $g$ is a constant vector field the conclusion follows easily by successive approximations. For arbitrary $f, g$ on $\mathbf{R}^{n}$, one can find $\bar{f}, \bar{g}$ on $\mathbf{R}^{n+1}$ and a submersion $F$ from $\mathbf{R}^{n+1}$ onto $\mathbf{R}^{n}$, with the property that $F_{*} \bar{f}=f, F_{*} \bar{g}=g$ and that, moreover, $\bar{g}$ is constant. This reduces the general case to the particular case $g=$ constant, and proves the theorem.

There is also a local theorem, in which $f$ and $g$ are defined on an open set $V \subseteq \mathbf{R}^{n}$, and only (1) and (2) are assumed. The conclusion in this case is that solutions exist and are unique on some interval which contains $t_{0}$ in its interior relative to $[a, b]$.

Theorem 1 makes it possible to pursue the "obvious approach" discussed before to define solutions of (stoch). If $f$ and $g$ satisfy the assumptions of Theorem 1 , and if the process $W=\left\{W_{t}: t \in[a, b]\right\}$, defined on the probability space $P=(\Omega, A, P)$ has continuous sample paths, then for every $t_{0}$ in $[a, b]$ and every random variable $\bar{X}$ on $P$ there exists a unique solution $X=\left\{X_{t}: t \in\right.$ $[a, b]\}$ of (stoch) such that $X\left(t_{0}\right)=\bar{X}$.

THEOREM 2. If $W$ is a Wiener process, then the solution of (stoch) in the sense of our definition coincides with the Stratonovich solution.

One can also construct solutions of (stoch) even when $f$ and $g$ do not satisfy assumptions (3) and (4) of Theorem 1. In this case, the solution will be defined, for each $\omega \in \Omega$, on an interval $I(\omega)$ which contains $t_{0}$ and is such that its endpoints are measurable functions of $\omega$.

REMARK. A result similar to Theorem 1 is definitely not true for equations $d x=f(x) d t+g_{1}(x) d w_{1}+\cdots+g_{k}(x) d w_{k}$, if the number $k$ of inputs is larger than one, as can be shown by simple examples. 


\section{REFERENCES}

1. D. Elliott, Controllable nonlinear systems driven by white noise, Unpublished doctoral dissertation, U.C.L.A., 1969.

2. J. Lamperti, A simple construction of certain diffusion processes, J. Math. Kyoto Univ. 4 (1964), 161-170. MR 31 \#808.

DEPARTMENT OF MATHEMATICS, RUTGERS UNIVERSITY, NEW BRUNSWICK, NEW JERSEY 08903 